\title{
SAÚDE OCUPACIONAL E ENFERMAGEM: ALGUMAS CONSIDERAÇÕES
}

Maria Helena Palucci Marziale* Emília Campos de Carvalho* Ana Emilia Pace Ferraz* Hujiko Enoki*

\begin{abstract}
RESUMO - Buscando identificar produção científica sobre saúde ocupacional relacionada à Enfermagem, foram revistos seis periódicos nacionais divulgados nos últimos 18 anos. Os artigos foram classificados segundo a área de atuação do autor, fonte, ano da publicação, modelo metodológico e enfoques quanto as atribuições do enfermeiro do trabaIho. Os resultados mostram que: os autores atuam tanto no campo docente como no assistencial; as metodologias científicas mais utilizadas foram reflexão teórica, exploratória e quantitativo descritiva; abordam predominantemente a assistência à clientela e organização do serviço de enfermagem do trabalho.
\end{abstract}

\begin{abstract}
The objective of the present study was to survey 6 Brazilian journals published over the last 18 years in order to identify scientific production on occupational health related to Nursing. The articles were classified by author's area of activity, source, year of publication, methodological model and attributes of nurses involved in occupational health. The results showed that authors act both in the teaching and assistance areas; the scientific methodologies most often used were theoretical reflection, exploratory and quantitative descriptive; the subjects most often approached were assistance to clients and organization of occupational nursing services.
\end{abstract}

\section{INTRODUC̣ÃO}

A Enfermagem na Área de Saúde Ocupacional começou a moldar-se, comolembra LEIFERT (1984), através das Portarias no 3.236 e 3.237 do Ministério do Trabalho, que tornavam obrigatórios serviços de saúde ocupacional nas empresas com mais de 100 empregados. Em 1975, através da Portaria no 3.460, o enfermeiro do trabalho foi reconhecido como parte integrante da equipe de saúde ocupacional nas empresas e logo a seguir através da Norma Regulamentadora (NR-4) de 1978 alterada em 1983 pelas Portarias no 33 e 34, as empresas com número de empregados superior a 3.500 passam a ser obrigadas a possuir enfermeiro no seu quadro de pessoal, com jornada de trabalho variável de acordo com o grau de risco da empresa.

No que compete à formação de recursos humanos para este campo, salienta-se o primeiro curso de espe- cialização para enfermeiro do trabalho, que foi realizado em 1975 pela Escola Paulista de Enfermagem em convênio com a FUNDACENTRO (LEIFERT 1984).

São várias as atribuições propostas ao enfermeiro do trabalho dos serviços de saúde ocupacional. Dentre elas, para NOGUEIRA (1982), estão inclusos a participação do planejamento, execução e avaliação de programas de saúde para os trabalhadores por meio de atuação técnico-administrativa, prestação de assistência direta ou da delegação e supervisão da assitência prestada por pessoal auxiliar; ensino e pesquisa na área de enfermagem do trabalho para proteção e recuperação da saúde do trabalhador.

Embora a legislação determine a obrigatoriedade do profissional nas empresas, as suas atribuições nem sempre estão sendo desempenhadas, pois, o enfermeiro muitas vezes atua conforme as exigências da empresa onde ele trabalha, além do que, deparam-se também

\footnotetext{
* Docente do Departamento de Enfermagem Geral e Especializada da Escola de Enfermagem de Ribeirão Preto da Universidade de São Paulo.
} 
com a falta de conhecimento de sua formação profissional e ainda encontram dificuldades em relação as definições de suas atribuições profissionais.

Estas indefinições quanto a prática da Enfermagem não estão restritas à especialização na área de saúde ocupacional. Também nos demais setores de sua atuação estes problemas ocorrem.

Segundo MENDES \& TREVIZAN (1983), depara-se com uma prática de enfermagem complexa com diversas categorias realizando atividades num emaranhado de indefinições, com estruturas teóricas diferentes na sua própria constituição, falta de conhecimentos teóricos que direcionem a prática, não entendimento do que seja a Enfermagem para grande parte da sociedade, falta de consenso como profissional liberal são fatos que levam à reflexão de que a Enfermagem como ciência nesse sentido ainda está adolescendo.

Frente à complexidade do quadro de indefinições acerca deste profissional e buscando conhecer o que vem sendo publicado a este respeito, vinculado às atribuições do enfermeiro do trabalho, foi elaborado o presente estudo.

Para execução deste levantamento teve-se como referencial os trabalhos sobre análise da produção científica desenvolvidos por VIEIRA (1980), NOGUEIRA (1982), ALMEIDA et alii (1981), NEVES (1982) e MENDES \& TREVIZAN (1983).

\section{São objetivos deste trabalho:}

- Analisar a produção científica de enfermeiros na área de saúde ocupacional;

- Identificar as atribuições mais relatadas pelos autores estudados.

\section{METODOLOGIA}

Foram consultadas para o presente estudo as publicações existentes a partir de 1968 até 1985 nos seguintes periódicos: Revista Brasileira de Saúde Ocupacional, Revista Brasileira de Enfermagem, Revista Enfermagem em Novas Dimensões, Revista Enfermagem Atual, Revista Enfermagem Moderna e Revista Paulista de Enfermagem.

Os critérios para inclusão dos trabalhos foram:

- ser periódico nacional;

- ser de autor ou co-autor enfermeiro;

- conter temas pertencentes a área de saúde ocupacional.

Os artigos foram classificados e analisados através de formulário contendo:

- identificação do periódico e ano de publicação;

- área de atuação do autor: assistencial, docência, docente-assistencial, administração geral e outros;

- área de predomínio do trabalho segundo as atribuições do enfermeiro do trabalho relatadas por NOGUEIRA (1982);

- metodologia de pesquisa utilizada: exploratório, ex- perimental, descritivo quantitativo, descritivo de caso e relato de experiência.

\section{RESULTADOS E DISCUSSÕES}

Com o intuito de verificar a existência de correlação entre publicações e momento histórico, iniciou-se o presente estudo a partir de 1968, embora, se saiba que só a partir de 1975 o enfermeiro vem fazendo parte integrante da equipe de saúde ocupacional. Esta preocupação encontra respaldo em SPERA (1958) ao comentar que a Enfermagem acompanha o desenvolvimento das ciências e o progresso do país. Desde esta época a autora já salientava os reflexos do desenvolvimento da industrialização na educação em Enfermagem, propondo nos currículos a introdução de higiene industrial.

Os dados obtidos (Tabela 1) parecem sugerir uma incipiente preocupação com os problemas relacionados a saúde do trabalhador no periodo de 1968 a 1975, uma vez que duas publicações foram encontradas.

0 incremento nas publicações se deu no período de 1976 a 1980, sendo encontrados nove artigos, provavelmente devido à recém regulamentação do enfermeiro na equipe de saúde ocupacional neste período. Nos anos seguintes (1981 a 1985), foram publicados sete artigos.

No periodo estudado foram encontrados 18 artigos, evidenciando a necessidade de maior número de estudos nesta área, buscando fornecer subsídios para embasamento do conhecimento.

Sabendo das dificuldades que o enfermeiro do trabalho ainda encontra para firmar seu espaço profissional, pode-se questionar: não poderia ser a pesquisa sobre enfermagem em saúde ocupacional um instrumento facilitador para este processo?

As dificuldades dos enfermeiros em fazer pesquisa na área de saúde ocupacional parecem estar ligadas, assim como nos demais setores da Enfermagem, à: - dificuldade para publicação;

- desconhecimento das empresas sobre a necessidade de pesquisa na área, para desenvolvimento da mesma;

- desconhecimento por parte dos enfermeiros sobre metodologia de pesquisa e pequeno destaque voltado a área de saúde ocupacional pelas escolas de Enfermagem.

Os periódicos que trouxeram maior número de publicações em saúde ocupacional foram: Revista Brasileira de Enfermagem ( 9 artigos); Revista Brasileira de Saúde Ocupacional (6 artigos); Revista Paulista de Enfermagem (2 artigos); Enfermagem em Novas Dimensões (1 artigo) e os periódicos Enfermagem Moderna e Enfermagem Atual não publicaram trabalhos pertencentes a este tema segundo o critério adotado. 
TABELA 1 - Distribuição de publicações sobre Enfermagem em Saúde Ocupacional segundo ano de publicação, periódico, área de atuação do autor e metodologia utilizada.

\begin{tabular}{|c|c|c|c|c|c|c|}
\hline \multirow{2}{*}{$\begin{array}{c}\text { ANO DE } \\
\text { PUBLICAÇÃO }\end{array}$} & \multicolumn{6}{|c|}{ PERIÓDICO } \\
\hline & $\begin{array}{c}\text { REV. BRAS. DE } \\
\text { SAÚDE OCUPACIONAL }\end{array}$ & $\begin{array}{l}\text { REV. BRAS. DE } \\
\text { ENFERMAGEM }\end{array}$ & $\begin{array}{c}\text { REV. ENF. EN } \\
\text { NOVAS DIMENSŌES }\end{array}$ & $\begin{array}{l}\text { REV. ENF. } \\
\text { MODERNA }\end{array}$ & $\begin{array}{l}\text { REV. ENF. } \\
\text { ATUAL }\end{array}$ & $\begin{array}{l}\text { REV. PAULISTA } \\
\text { DE ENFERMAGEM }\end{array}$ \\
\hline \multicolumn{7}{|l|}{ 1968-1971 } \\
\hline 1972 & & 1 & & & & \\
\hline \multicolumn{7}{|l|}{ 1973-1974 } \\
\hline 1975 & & 1 & & & & \\
\hline 1976 & & 2 & & & & \\
\hline 1977 & 2 & 2 & & & & \\
\hline 1978 & & 1 & 1 & & & \\
\hline \multirow{2}{*}{\multicolumn{7}{|c|}{1980}} \\
\hline & & & & & & \\
\hline 1981 & & & & & & 1 \\
\hline 1982 & & 1 & & & & \\
\hline 1983 & 2 & & & & & \\
\hline 1984 & 1 & & & & & 1 \\
\hline 1985 & 1 & & & & & \\
\hline TOTAL & 6 & 9 & 1 & 0 & 0 & 2 \\
\hline
\end{tabular}

\begin{tabular}{|c|c|c|c|c|c|}
\hline \multirow{2}{*}{$\begin{array}{c}\text { ANO DE } \\
\text { PUBLICAÇÃO }\end{array}$} & \multicolumn{5}{|c|}{ ÁREA DE ATUAÇÃO DO AUTOR } \\
\hline & ASSISTENCIAL & DOCÊNCIA & $\begin{array}{c}\text { DOCENTE } \\
\text { ASSISTENCIAL }\end{array}$ & $\begin{array}{l}\text { ADMINISTRACĀO } \\
\text { GERAL }\end{array}$ & OUTROS \\
\hline \multicolumn{6}{|l|}{ 1968-1971 } \\
\hline 1972 & & & & 1 & \\
\hline \multicolumn{6}{|l|}{ 1973-1974 } \\
\hline 1975 & 1 & & & 1 & \\
\hline 1976 & 1 & & & & \\
\hline 1977 & 1 & 1 & & & 2 \\
\hline 1978 & & 2 & & & \\
\hline 1979 & 1 & & & & \\
\hline 1980 & & & & & \\
\hline 1981 & & 1 & & & \\
\hline 1982 & 1 & & & & \\
\hline 1983 & & 2 & & 1 & \\
\hline 1984 & 1 & & & & \\
\hline 1985 & & 2 & & & \\
\hline TOTAL & 6 & 7 & a & 3 & 2 \\
\hline
\end{tabular}




\begin{tabular}{|c|c|c|c|c|c|c|c|}
\hline \multirow{2}{*}{$\begin{array}{c}\text { ANO DE } \\
\text { PUBLICAÇÃO }\end{array}$} & \multicolumn{7}{|c|}{ METODOLOGIA } \\
\hline & $\begin{array}{c}\text { REFLEXÃO } \\
\text { TEÓRICA }\end{array}$ & EXPLORATÓRIA & EXPERIMENTAL & $\begin{array}{l}\text { QUANTITATIVO } \\
\text { DESCRITIVO }\end{array}$ & $\begin{array}{c}\text { DESCRITIVO } \\
\text { DE CASO }\end{array}$ & $\begin{array}{c}\text { RELATO } \\
\text { DE EXPERIÊNCIA }\end{array}$ & $\begin{array}{c}\text { COMUNICACÃO } \\
\text { DE CONFERÊNCIA }\end{array}$ \\
\hline \multicolumn{8}{|l|}{ 1968-1971 } \\
\hline 1972 & & & & & & 1 & \\
\hline \multicolumn{8}{|l|}{ 1973-1974 } \\
\hline 1975 & 1 & & & & & & \\
\hline 1976 & & 2 & & & & & \\
\hline 1977 & & 2 & & 1 & & & 1 \\
\hline 1978 & 2 & & & & & & \\
\hline 1979 & & & & & & 1 & \\
\hline \multicolumn{8}{|l|}{1980} \\
\hline 1981 & 1 & & & & & & \\
\hline 1982 & & & & 1 & & & \\
\hline 1983 & 1 & & & 1 & & & \\
\hline 1984 & 2 & & & 1 & & & \\
\hline 1985 & & & & 1 & & & \\
\hline TOTAL & 7 & 4 & 0 & 4 & 0 & 2 & 1 \\
\hline
\end{tabular}

Embora a Revista Brasileira de Saúde Ocupacional seja especializada no assunto, considerou-se pequeno o número de artigos de autores ou co-autores enfermeiros. Quanto a maior incidência das publicações ser da Revista Brasielira de Enfermagem, atribui-se talvez ao fato de ser a mais tradicional e possuir maior número de perioódicos publicados.

Observando ainda a Tabela 1 , nota-se quanto às áreas de atuação dos autores que a docente (7) e assistencial (6) se assemelham. Salienta-se porém que dentre os artigos inclusos na área assistencial 2 são de coautores enfermeiros e não de autores enfermeiros.

Esta equivalência pode estar relacionada com o cretério da categorização utilizando no presente estudo que leva em conta a descrição da identificação feita pelo autor nos artigos. Desta forma 3 artigos foram classificados pertencentes a área de administração geral, dado os critérios citados, embora sabe-se que seus autores também mantêm atividades de docência em Enfermagem ainda que não a mencionassem.

$\mathrm{Na}$ categoria outros, encontra-se um artigo publicado por presidente de instituição de classe e um por aluno. Não observou-se publicações ef etuadas por autores que se identificassem na área docenteassistencial; também aqui este achado pode estar relacionado ao fato de se considerar como área de atuação do autor apenas a primeira descrita na apresentação do artigo.

Quanto ao tipo de metodologia utilizada, visualizase na Tabela 1 , um predomínio dos trabalhos de reflexão teórica, o que demonstra que os autores têm optado por um dos tipos metodológicos mais simples, uma vez que caracteriza-se por aqueles de exposição de um tema numa argumentação e interpretação pessoal.

Encontra-se também quatro artigos que utilizam a metodologia exploratória, caracterizados por articu- lação de conceitos na formulação de um problema procurando descobrir novas relações, pode ser considerado como primeiro passo no processo de pesquisa.

Quatro artigos utilizam o tipo quantitativo descritivo, descrevendo uma situação levando em conta o estudo de poucas variáveis em grande número de casos, podendo ou não formular hipóteses.

Observa-se ainda dois artigos que relatam experiência na prática de Enfermagem em Saúde Ocupacional. Um artigo não se aplicou nenhum tipo metodológico por se tratar de comunicação de conferência. Não verificou-se artigo abordado através de metodologia de trabalho experimental, que é aquele destinado a testar hipóteses específicas que envolvem relações de causa e efeito; e nem trabalho usando a metodologia descritiva de caso, que caracteriza-se por descrever uma situação levando em conta o estudo de grande número de variáveis em um caso ou em um pequeno número de casos, podendo ou não formular hipóteses.

Esses dados evidenciam uma concordância aos achados de MENDES \& TREVIZAN (1983), quando analisam a utilização do Método Científico em Enfermagem, encontrando predominantemente as formas mais simples de pesquisas.

Os temas dos artigos observados foram também categorizados através da identificação de atribuições do enfermeiro do trabalho dos serviços de saúde ocupacional, mencionados por NOGUEIRA (1982). Esta tentativa visou detectar quais atribuições que predominam nas publicações e estão apresentadas na Tabela 2 .

Observou-se que os autores detiveram-se nas atribuições de planejamento da assistência à clientela $\mathrm{e}$ prestação dos cuidados mais complexos (6 artigos); seguido do planejamento e organização do serviço de enfermagem do trabalho (4 artigos). 
TABELA 2 - Distribuição das Publicações sobre Enfermagem em Saúde Ocupacional segundo as atribuições do enfermeiro.

\begin{tabular}{|c|c|c|c|c|c|c|c|c|c|c|c|c|c|}
\hline & ATO DE PUBLICAÇÃO & & & & & & & & & & & & \\
\hline $\begin{array}{l}\text { IDENTIFICAÇÃO } \\
\text { DE ATRIBUIÇŌES }\end{array}$ & & 1974 & 1975 & 1976 & 1977 & 1978 & 1979 & 1981 & 1982 & 1983 & 1984 & 1985 & TOTAL \\
\hline
\end{tabular}

Coletar e analisar junto com a equipe de saúde ocupacional os dados sócio-sanitários da população de empregados a ser atendido pelo serviço de saúde ocupacional da empresa.

Elaborar com a equipe normas técnicas e administrativas para o serviço.

1

Estabelecer com a equipe, o programa de saúde para os empregados da empresa.

Coletar e analisar os dados referentes às necessidades da enfermagem do programa e recursos materiais e humanos existentes na empresa e comunidade.

Planejar e organizar o serviço de enfermagem do trabalho.

Planejar a assistência à clientela e prestar os cuidados mais complexos

Delegar os cuidados menos complexos ao auxiliar de enfermagem e supervisionar os cuidados que foram planejados por ele.

Planejar, executar e avaliar programa de educação a saúde para os empregados.

Participar juntamente com a equipe de outros departamentos quando visam a saúde do trabalhador.

Participar no ensino de enfermagem do trabalho e na supervisão de estagiários.

Planejar, executar e avaliar pesquisa em enfermagem do trabalho e participar de pesquisas.

Outros
1

\begin{tabular}{lllllllllllllll}
\hline TOTAL & 1 & 1 & 2 & 4 & 2 & 1 & 1 & 1 & 2 & 2 & 1 & 18 \\
\hline
\end{tabular}

Ainda foram identificadas as atribuições de coletar e analisar dados referentes às necessidades da Enfermagem do programa e recursos materiais e humanos existentes na empresa e comunidade, ( 2 artigos) e o de estabelecer com a equipe, o programa de saúde para os empregados da empresa (2 artigos); a atribuição de coletar e analisar juntamente com a equipe de saúde ocupacional os dados sócio-econômicos e sanitários da população de empregados a serem atendidos pelo serviço de saúde ocupacional da empresa, também foi abordado (1 artigo); assim como a de elaboração com a equipe de normas técnicas e administrativas para o serviço (1 artigo).
Considerou-se também o estudo das condições de trabalho do pessoal de enfermagem (1 artigo), embora essa não seja um das atribuições descritas por NOGUEIRA (1982).

Cumpre ressaltar que a atividade de planejar, executar, avaliar e participar de pesquisa em Enfermagem, está implícita também nas atribuições dos enfermeiros assistenciais. Portanto os seis artigos dos autores que têm esta área de atuação, poderão ser considerados pertencentes a esta casela; a temática destes estudos sugerem o que está sendo pesquisado neste campo.

Existem algumas lacunas em atribuições que não 
foram abordadas e estudadas até o momento, são elas:

- delegação dos cuidados menos complexos ao auxiliar de enfermagem e supervisionar os cuidados que foram planejados por ele;

- planejamento, execução e avaliação de programa de educação e saúde para os empregados;

- participação juntamente com a equipe de programas de outros departamentos quando visam a saúde do trabalhador;

- participação no ensino de Enfermagem do trabalho e na supervisão de estagiários.

Trabalhos nestas áreas, poderão ser realizados para 0 avanço do conhecimento na área de Enfermagem em saúde ocupacional.

\section{CONSIDERAÇÕES FINAIS}

No presente estudo foram relevantes os seguintes achados:

- é incipiente o número de trabalhos publicados nesta área;

- os periódicos que apresentam maior número de publicações são Revista Brasileira de Enfermagem e a Revista Brasileira de Saúde Ocupacional;

- a área de atuação de autor se assemelham entre docência e assistencial;

- a metodologia mais utilizada é a reflexão teórica e assistencial;

- os enfermeiros estão mais prøocupados em publicar sobre atividade de planejar a assistência à clientela e organizar o serviço de enfermagem do trabalho.

Diante do exposto, a área de saúde ocupacional vem se constituindo um desafio para a Enfermagem; parece necessário pesquisar e estudar os riscos provenientes do trabalho executado, conhecer o material utilizado, as condições de segurança e higiene proporcionadas, visando manter o trabalhador hígido para a execução de suas funções.

\section{REFERÊNCIAS BIBLIOGRÁFICAS}

1. ALMEIDA, M.C.P. de et alii. A produção do conhecimento na pós-graduação em Enfermagem no Brasil. In: CONGRESSO BRASILEIRO DE ENFERMAGEM, 33, Manaus, 1981. Anais... p. 119-26.

2. MENDES, I.A.C. \& TREVISAN, M.A. Acerca da utilização do Método Científico nas pesquisas de Enfermagem. Revista Brasileira de Enfermagem. Brasília, 36(1):13-9, jan./fev. 1983.

3. NEVES, E.P. Vazios do conhecimento e sugestões relevantes na área de Enfermagem. In: SEMINÁRIO NACIONAL DE PESQUISA EM ENFERMAGEM, 2. Brasília, 1982. Anais...

4. NOGUEIRA, M.J. de C. Níveis de Prevenção e m Enfermagem do Trabalho. Revista da Escola de Enfermagem da USP, São Paulo, 16(3): 275-84, dez. 1982 a.

5. A pesquisa em Enfermagem no Brasil - retrospectiva histórica. In: SEMINÁRIO NACIONAL DE PESQUISA EM ENFERMAGEM, 2. Brasília, 1982 b. Anais..

6. Subsídios para a descrição d o conteúdo de ocupação enfermeiro de saúde pública. Revista Enfermagem em Novas Dimensões, São Paulo, 1(3):119-25, jul./ago. 1975.

7. SPERA, F.C. Enfermagem industrial - Formação e treinamento da Enfermeira. Revista Brasileira de Enfermagem, Rio de Janeiro 11(1):52-59, março. 1958.

8. VIEIRA, T.V. Produção Científica em Enfermagem no Brasil: 1960-1979. Salvador, Bahia, UFBA, 1980. Tese Prof. Titular. 